Wann ist der Mensch tot? Die grosse Bedeutung einer kleinen Frage

Monteverde, Settimio

Posted at the Zurich Open Repository and Archive, University of Zurich ZORA URL: https://doi.org/10.5167/uzh-103822

Journal Article

Published Version

Originally published at:

Monteverde, Settimio (2014). Wann ist der Mensch tot? Die grosse Bedeutung einer kleinen Frage. Bioethica Forum, 7(1):13-14. 


\title{
Wann ist der Mensch tot? Die grosse Bedeutung einer kleinen Frage
}

\author{
Settimio Monteverde \\ a Institut für Biomedizinische Ethik, Universität Zürich
}

Wann ist der Mensch tot? Obschon grammatikalisch einfach, fördert jeder Versuch, diese «kleine Frage» zu beantworten, die Verwobenheit biologischer, normativer, kultureller und sozialer Aspekte zutage, wenn es darum geht, sich dem Tod des Menschen begrifflichkonzeptuell und phänomenologisch zu nähern. Da «[...] jede Grenzziehung zwischen Leben und Tod kulturbedingt ist» [1], mag der Versuch, sie ausschliesslich aus dem «Buch der Natur» heraus beantworten zu wollen, zwar eine Vielzahl an notwendigen, aber dennoch keineswegs hinreichenden Antworten zu geben. Inwiefern das Vorhandensein gewisser Hirnfunktionen, endokriner oder elektrischer Aktivitäten bei diagnostiziertem Hirntod - wie in den letzten Jahren intensiv diskutiert - an dieser Grenzziehung etwas ändern sollen und können, ist eine Frage, die sich auf der Ebene des Befundes primär einmal beschreiben, nicht aber schon lösen lässt.

Obschon die Frage nach dem Tod des Menschen aus kulturgeschichtlicher Perspektive keineswegs neu ist, wird sie seit einem guten halben Jahrhundert durch den medizinisch-technischen Fortschritt in einer zuvor ungeahnten Radikalität aufgeworfen. Dieser Fortschritt zeigte sich in drei Kontexten medizinischen Handelns, die entstehungsgeschichtlich zunächst unabhängig voneinander $\mathrm{zu}$ betrachten sind, im Gefolge der Entwicklungen sich aber in erheblichem Masse gegenseitig bedingt haben: Erstens die diagnostische Möglichkeit der neurologischen Todesbestimmung, zweitens die klinische Suche nach einer zuverlässigen Grenze der ärztlichen Behandlungsverpflichtung unter den Rahmenbedingungen der Intensivmedizin [2] und drittens die Fortschritte in der Transplantationsmedizin, der dadurch entstehende relative Organmangel, die Verfügbarkeit von Organersatztherapien für Patientinnen und Patienten auf der Warteliste und die Möglichkeit des Organerhalts im Zustand des Hirntods [3].

Diese Kontext-Trias konstituiert eine Grundspannung, welche die Diskussion um den Tod des Menschen im Zusammenhang mit der Möglichkeit der neurologischen Bestimmung bis heute prägt: Liegt der Akzent der «kleinen Frage» auf dem Adverb wann, dann fokussiert die Fragestellung den Todeszeitpunkt. Speziell geht es hier um das Problem, ob der Tod nicht nur aposteriorisch im Zustande eines «Gesamttodes» erkennbar ist - d.h. bei Ausfall sämtlicher Organsysteme, die für das menschliche Leben notwendig sind -, sondern gewissermassen schon apriorisch, d.h. bei Ausfall nur eines Teils dieser Organsysteme, der aber für das Leben des Menschen für derart bedeutungsvoll betrachtet wird, dass dessen Ausfall als hinreichend sicheres Todeszeichen gilt. Liegt der Akzent hingegen auf dem Substantiv Mensch, stellt sich die Frage nach dem spezifischen Unterscheidungsmerkmal des Todes von Menschen im Unterschied etwa zum Tod von anderen Wirbeltieren, Mikroorganismen oder Pflanzen. Bereits hier wird deutlich, dass sich die Frage nach dem Tod des Menschen nicht ohne Rückgriff auf anthropologische Grundannahmen beantworten lässt. Festzulegen, von welchem Moment an ein Mensch aufhört, Teil einer sozialen, religiösen, rechtlichen und moralischen Gemeinschaft zu sein, ist für deren Erhalt von vitaler Bedeutung. Denn davon ausgehend werden Besitzrechte übertragen, Rentenansprüche an die Hinterbliebenen gewährt und Handlungen an den Verstorbenen vorgenommen, die zu Lebzeiten nicht durchführbar wären wie etwa Begräbnis, Kremation, Autopsie oder die Entnahme vitaler Organe. An diesen Implikationen wird die moralische Natur der Frage nach dem Tod des Menschen sichtbar [4]. Nicht zuletzt kann der Akzent auch auf das Adjektiv tot gelegt werden, womit, in einem aposteriorischen Sinne, ein Zustand bezeichnet ist, der sich empirisch nur negativ auf der Grundlage eines wie auch immer artikulierten - Lebensbegriffes ableitet und konstatiert, dass die für das Leben eines Menschen wesentlich erachteten Konnotationen nicht mehr gegeben sind, wie das Eduard Zwierlein etwa im Begriff des «geistiges Niemandslands» [5] ausdrückt. Die grosse Bedeutung der «kleinen Frage» zeigt sich also darin, dass sich aufgrund der zeitlichen Dissoziation des Sterbens unter den technisch-apparativen Bedingungen der Intensivmedizin und der oben erwähnten KontextTrias die alte Frage nach dem Übergang zwischen Leben und Tod radikal neu stellt. Die mit dem Tod des Menschen einhergehenden Veränderungen im moralischen Status sind nicht der Natur des Menschen unmittelbar zu entnehmen. Und dennoch braucht es wegen der moralischen Zäsur, die der Tod des Menschen für Individuum und Gemeinschaft bedeutet, empirische, der menschlichen Natur inhärente Korrelate, um den Eintritt des Todes mit an Sicherheit grenzender Gewissheit festzustellen. Diese Notwendigkeit ist es, welche die klinische Bedeutung der neurologischen Todesbestimmung ausmacht und die - von Missbräuchen in der Allokationspraxis relativ unabhängige - Akzeptanz von Fachpersonen erklärt [2]. Kritisch bleibt hier anzu- 
merken, dass eine verlässliche Datensammlung in dieser konkreten Frage ausgesprochen schwierig ist (Selektionsbias, unterschiedliche Kriterien der Todesbestimmung wie z.B. Hirnstammtod in Grossbritannien und Irland) $[6,7]$.

Ein Rückblick auf die aktuellen Debatten rund um die neurologische Definition des Todes, die durch das Weisspapier des US-President's Council von 2008 in eine neue Runde trat, bestätigt diese Einsicht [8]. Selbst in der Auslegung des Weisspapiers und in der Beurteilung der Frage, wie valide das klassische Ganzhirntodkriterium auch in seinen Fortschreibungen ist, scheiden sich die Geister in zwei unversöhnliche Lager, die von ihren Grundannahmen ausgehend das Glas als halbleer oder halbvoll betrachten. Im Blick auf die Bedeutung der Todesbestimmung in der Klinik und im Umgang mit Patienten, Angehörigen und Fachpersonen stellen sich verschiedene Probleme: So zum Beispiel, welche Rolle den Medien in der Berichterstattung zukommt, etwa wenn es z.B. heisst: «Die hirntote Frau wird nur noch durch Infusionen am Leben erhalten» [9], ferner, ob eine «responsible scholarship» der Forschenden nur solche Kritik zulassen sollte, die das öffentliche Vertrauen in die Ärzteschaft nicht erodiert [10], oder aber, ob eine solche «Selbstzensur» seriöse Forschung nicht kompromittiere und es vielmehr geboten sei, relevante Fakten an die Öffentlichkeit $\mathrm{zu}$ bringen [11]. Wie auch immer der Nexus zwischen philosophischen, klinischen, wissenschafts- und berufsethischen Aspekten vollzogen wird: Es ist unrealistisch, davon auch ein Ende der Debatte zu erwarten. Ebenso wenig sind Lösungen dienlich, die sozial nicht adäquat sind, auf einer abstrakt-theoretischen Ebene bleiben und die Diskussion frühzeitig abbrechen, so etwa ein Verzicht auf eine neurologische Definition des Todes in der Transplantationsmedizin gekoppelt mit der Aufgabe der Dead Donor Rule und der Einführung einer engen Zustimmungslösung [12].

Dass es einen Dissens hinsichtlich der Beantwortung der «kleinen Frage» nach dem Tod des Menschen gibt, zeigt sich heute erneut mit aller Deutlichkeit. Die nächste Frage, die sich angesichts dieses Status quo stellt, ist, ob nach 50 Jahren Debatte wenigstens von einem begründeten Dissens gesprochen werden kann. Doch da kommen Zweifel auf, denn begründet wäre er dann, wenn die Gründe auch offenliegen würden, was bedingt, dass Befürworter wie auch Gegner ihre anthropologischen Grundannahmen konsequent darlegen und auf ihre Plausibilität hin erörtern. Diese würden vermutlich eine antinaturalistische und eine stark naturalistische Position in der Frage des Todes des Menschen offenlegen. Neben diesen zwei epistemologischen Wegen, den Tod des Menschen zu denken, schlägt
Eric Racine eine dritte Möglichkeit vor, das Verhältnis von Bioethik und Natur zu beschreiben, nämlich einen moderat pragmatischen Naturalismus [13]. Letzterer könnte in der Lage sein, die Bedeutung der empirischen Befunde, die die Kritik an der rezipierten Hirntodkonzeption prägen, in ein Licht zu rücken, das die Relevanz dieser Befunde nicht schmälert, aber ebenso wenig den ethisch-juristischen und klinischen Konsens, der sich in der Hirntodkonzeption gebildet hat.

\section{Korrespondenz}

Settimio Monteverde, lic. theol. MAE, MME, RN

Institut für Biomedizinische Ethik

Universität Zürich

Pestalozzistrasse 24

$\mathrm{CH}-8032$ Zürich

E-Mail: settimio.monteverde[at]uzh.ch

\section{Referenzen}

1 Schlich, T. (2001). Tod, Geschichte, Kultur. In T. Schlich, \& C. Wiesemann, Hirntod. Zur Kulturgeschichte der Todesfeststellung (S. 9-42). Frankfurt a.M.: Suhrkamp.

2 Söffker, G., Bhattarai, M., Welte, T., Quintel, M., \& Kluge, S. (2013). Einstellung des intensivmedizinischen Fachpersonals zur postmortalen Organspende in Deutschland. Medizinische Klinik - Intensivmedizin und Notfallmedizin, S. dx.doi.org/10.1007/s00063-013-0271-x.

3 Rhodes, R., \& Schiano, T. (2012). Justice in transplant organ allocation. In R. Rhodes, M. P. Battin, \& A. Silvers, Medicine and social justice. Essays on distribution of health care (2. Ausg., S. 505-521). Oxford: Oxford University Press.

4 DeGrazia, D. (Fall 2011). The definition of death. (E. N. Zalta, Hrsg.) Abgerufen am 19.11.2013 von The Stanford Encyclopedia of Philosophy: http://plato.stanford.edu/archives/fall2011/entries/deathdefinition/

5 Zwierlein, E. (2012). Der Hirntod und der Tod des Menschen. In H. Niederschlag, \& I. Proft (Hrsg.), Wann ist der Mensch tot? Diskussion um Hirntod, Herztod und Ganztod (Ethische Herausforderungen in Medizin und Pflege. Ausg., Bd. 3, S. 19-30). Ostfildern: Mathias Grünewald

6 Joffe, A.R., Anton, N.R., Duff, J.P., deCaen, A (2012). A survey of American neurologists about brain death: understanding the conceptual basis and diagnostic tests for brain death. Annals of Intensive Care (2) S.1-8.

7 Ledger, U.S., Begley, A., Reid, J., Prior, L., McAuley, D., Blackwood, B. (2013). Moral distress in end-of-life care in the intensive care unit. Journal of Advanced Nursing (69) S. 1869-1880.

8 President's Council on Bioethics (2008). Controversies in the determination of death. A White Paper of the President's Council on Bioethics. The President's Council on Bioethics: Washington, DC. http://bioethics.georgetown.edu/pcbe/reports/death/index.html Abruf: 22.11.2013

9 Daoust, A., Racine, E. (2013). Depictions of 'brain death' in the media: medical and ethical implications. J Med Ethics. Published Online First: 12 April 2013 doi:2010.1136/medethics-2012-101260.

10 DuBois, J. M. (2010). The ethics of creating and responding to doubts about death criteria. J Med Philos (35) S. 365-380.

11 Potts M, Verheijde D E, Rady M, Evans D (2013). The ethics of limiting informed debate: Censorship of select medical publications in the interest of organ transplantation. Journal of Medicine and Philosophy (38) S. 625-638.

12 DuBois, J. M. (2011). Dead tired of repetitious debates about death criteria. American Journal of Bioethics (11) S. 45-47.

13 Racine, E. (2008). Which naturalism for bioethics? A defense of moderate (pragmatic) naturalism. Bioethics, 22, S. 92-100. 